\title{
Noninvasive Assessment of Spatio-Temporal Recurrence in Atrial Fibrillation
}

Citation for published version (APA):

Bonizzi, P., Zeemering, S., Rosmalen, F. V., Schotten, U., \& Karel, J. (2021). Noninvasive Assessment of Spatio-Temporal Recurrence in Atrial Fibrillation. In 2020 28th European Signal Processing Conference (EUSIPCO) (pp. 900-904). [9287452] IEEE Xplore. https://doi.org/10.23919/Eusipco47968.2020.9287452

Document status and date:

Published: 21/01/2021

DOI:

10.23919/Eusipco47968.2020.9287452

Document Version:

Publisher's PDF, also known as Version of record

Document license:

Taverne

Please check the document version of this publication:

- A submitted manuscript is the version of the article upon submission and before peer-review. There can be important differences between the submitted version and the official published version of record.

People interested in the research are advised to contact the author for the final version of the publication, or visit the DOI to the publisher's website.

- The final author version and the galley proof are versions of the publication after peer review.

- The final published version features the final layout of the paper including the volume, issue and page numbers.

Link to publication

\footnotetext{
General rights rights.

- You may freely distribute the URL identifying the publication in the public portal. please follow below link for the End User Agreement:

www.umlib.nl/taverne-license

Take down policy

If you believe that this document breaches copyright please contact us at:

repository@maastrichtuniversity.nl

providing details and we will investigate your claim.
}

Copyright and moral rights for the publications made accessible in the public portal are retained by the authors and/or other copyright owners and it is a condition of accessing publications that users recognise and abide by the legal requirements associated with these

- Users may download and print one copy of any publication from the public portal for the purpose of private study or research.

- You may not further distribute the material or use it for any profit-making activity or commercial gain

If the publication is distributed under the terms of Article $25 \mathrm{fa}$ of the Dutch Copyright Act, indicated by the "Taverne" license above, 


\section{Noninvasive Assessment of Spatio-Temporal Recurrence in Atrial Fibrillation}

\author{
Pietro Bonizzi \\ dept. of Data Science and Knowledge Engineering \\ Maastricht University \\ Maastricht, The Netherlands \\ pietro.bonizzi@maastrichtuniversity.nl
}

\author{
Stef Zeemering \\ dept. of Physiology \\ Maastricht University \\ Maastricht, The Netherlands \\ s.zeemering@maastrichtuniversity.nl
}

Joël Karel

dept. of Data Science and Knowledge Engineering Maastricht University

Maastricht, The Netherlands

joel.karel@maastrichtuniversity.nl

\begin{abstract}
Propagation of Atrial Activity during atrial fibrillation (AF) is a complex phenomenon characterized by a certain degree of recurrence (periodic repetition). In this study, we investigated the possibility to detect recurrence noninvasively from body surface potential map recordings in patients affected by persistent $\mathrm{AF}$, and localize this recurrence both in time and space. Results showed that clusters of recurrence can be identified from body surface recordings in these patients. Moreover, the number of clusters detected and their location on the topright of the back of the torso were significantly associated with AF recurrence 4 to 6 weeks after electrical cardioversion. This suggests that noninvasive quantification of recurrence in persistent AF patients is possible, and may contribute to improve patient stratification.
\end{abstract}

Index Terms-Atrial fibrillation, Noninvasive, Recurrence analysis, AF substrate complexity.

\section{INTRODUCTION}

Wave front propagation during atrial fibrillation (AF) is a complex phenomenon, which may be characterized by quasiperiodic patterns localized both in time and space. These propagation patterns may be driven by specific regions within the atria that spontaneously generate electrical activity in an uncoordinated way (repetitive focal activity [1]), or by selfperpetuating quasi-periodic re-entry patterns of propagation of electrical wave fronts, which may happen both globally (multiple wavelets at the level of the whole atria, [2], [3]), or locally (rotor activity at the level of sub-regions of the atria, [4]). Therefore, AF is characterized by recurrent mechanisms which may be localized both in time and space. This suggest that a natural way to characterize properties of wave front propagation patterns during $\mathrm{AF}$ is by means of tools for the investigation of recurrence in dynamical systems [5]. In this respect, it was shown in [6] that recurrence plots and recurrence quantification analysis are suitable for identifying sites of stable and repeatable bipolar electrogram morphology patterns, and it was suggested that sites with rapid activation of highly repetitive morphology patterns may be critical to sustaining AF. Zeemering et al. [7] used recurrence plot analysis combined with Principal Component Analysis (PCA) to build a reliable tool to visualize dynamical behaviour and to assess the complexity of wave front patterns in AF, based on unipolar electrograms.

Recurrence analysis is important also in terms of quantification of AF substrate complexity, which may form the basis for a more adequate AF stratification and may provide useful information for guiding AF therapy [8]. Indeed, previous studies have shown that ECG-based parameters such as quantifying $\mathrm{AF}$ organization can be used to determine $\mathrm{AF}$ substrate complexity [9]-[11]. However, these parameters try to capture global spatiotemporal and frequency properties of $\mathrm{AF}$ propagation patterns and may not sufficiently represent the continuous spectrum of AF progression. Therefore, these parameters may fail to properly characterize patients that have a similar AF substrate, but who may still respond differently to the same treatment. Hence, characterization of recurrence in AF propagation patterns may complement the information provided by the aforementioned parameters, and help their clinical applicability for AF diagnosis and treatment. In recent studies, we showed that propagation of atrial activity during $\mathrm{AF}$ is a process characterised by different short- and longterm recurrent behaviours [12], and that this could be used to investigate the origin of the time-varying spatio-temporal properties of AA propagation during AF [13].

Recently, we proposed a method to detect spatio-temporal recurrence in complex dynamical systems characterized by repetitive (quasi-periodic) oscillatory patterns [14]. We showed that, when applied to actual invasive recordings of atrial activity during AF in an animal model, the proposed framework was able to unveil regions on the atrial walls characterized by recurrent behaviors, which were not visible from the recurrent plots generated from the full available spatiotemporal data. This is relevant, as an accurate spatial and temporal identification of those regions may help characterize 


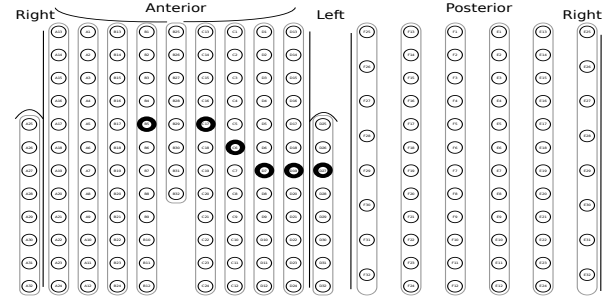

Fig. 1: BSPM electrode configuration, comprising 120 anterior leads and 64 posterior leads. Thick circles: default positions of the precordial leads $V_{1}, \ldots, V_{6}$.

the progression of the disease, the level of impairment of the cardiac tissue, and improve patients stratification and personalize therapy [11]. In this study, we employed this framework to explore the possibility of detecting recurrence of AF propagation patterns noninvasively from body surface potential map (BSPM) recordings. We propose a set of indices to characterize the identified regions of recurrence on the body surface, and assess their association with $\mathrm{AF}$ recurrence 4 to 6 weeks after electrical cardioversion. Preliminary results suggest that noninvasive characterization of recurrence of $\mathrm{AF}$ propagation patterns is possible, and is associated with $\mathrm{AF}$ recurrence after electrical cardioversion.

\section{Methods}

\section{A. BSPM data and pre-processing}

In this study, we employed a dataset of body surface potential maps (BSPMs) recorded in 75 consecutive patients in persistent AF (retrieved from Maastricht University Medical Centre, Maastricht, the Netherlands; the study was approved by the institutional ethics review board). BSPMs were recorded with 120 anterior and 64 posterior leads (ActiveTwo BSM Panels Carbon Electrodes, Biosemi B.V., The Netherlands) as shown in Fig. 1. All patients underwent electrical cardioversion, and 32 out of 63 patients with follow-up showed $\mathrm{AF}$ recurrence 4 to 6 weeks after procedure. ECGs were sampled at $2048 \mathrm{~Hz}$, and downsampled to $256 \mathrm{~Hz}$. A oneminute segment was selected for each subject, the low-quality leads were excluded (low signal-to-noise ratio, poor electrode contact, motion artefacts), and Wilson's Central Terminal was subtracted in line with conventional ECG analysis. All signals were band-pass filtered between 1 and $100 \mathrm{~Hz}$ (3rd order Chebyshev), and QRST cancellation was performed using an adaptive singular value decomposition method, inspired by the approach in [15], with multiple QRST window templates defined using hierarchical clustering. The extracted Atrial Activity (AA) signals were post-filtered with a zero-phase notch filter at $50 \mathrm{~Hz}$ (2nd order IIR filter) to suppress power line noise, and with a $3 \mathrm{~Hz}$ zero-phase high-pass filter (3rd order Chebyshev) to remove low-frequency residuals not related to (persistent) AF. One patient was excluded from the analyses because of a different lead configuration used to acquire the BSPM recordings.

\section{B. Spatio-temporal detection of recurrence}

The method used in this study to detect spatio-temporal recurrence was extensively described in [14]. Briefly, we assume to be dealing with a complex dynamical system characterized by an arbitrary geometric structure with $n$ points; at each point we have a uni-variate state variable that varies with time. We also assume that the geometric relationships among those points are well-defined (for instance, in terms of proximity: for each point, its neighboring points are specified). Each point in the geometry is characterized by a signal or a time series, which describes the evolution over time of the state variable associated with that point (e.g., a set of BSMP leads and their corresponding ECG recordings). In this context, recurrence can be defined in terms of spatial patterns that repeat over time. Such patterns may occur in specific regions of the structure (a person's torso in our case), and over limited time intervals.

The first step is to identify the most relevant points in the geometric structure associated with a specific recurrent pattern. This is achieved by first generating a matrix $B=(n \times N)$, which collects the signals from all points in the structure ( $n$ points having $N$ samples each). Matrix $B$ is then decomposed by means of PCA. The rationale for using PCA is the assumption that spatio-temporal repetitive quasi-periodic patterns are expected to be associated with highly correlated signals at different points of the structure, and this redundancy is expected to be picked-up by the first Principal Components (PCs) of $B$.

The second step is to identify how many PCs of $B$ are required to properly determine a recurrent pattern in the structure. For this, the most relevant PCs associated with a recurrent pattern are found by looking at their normalized power spectrum and by measuring the spectral concentration (SC) around their dominant frequency peaks. The most relevant PCs are identified as those whose SC is larger than a predefined threshold. The rationale is that recurrence associated with repetitive (quasi-periodic and oscillating) patterns should show clear peaks in the power spectrum of the corresponding PCs.

Once all relevant PCs of a recurrent pattern have been selected, the third step is to identify all points in the original geometric structure associated with those PCs, i.e., the points on which the selected PCs are reflected the most. For each PC, the most relevant points can be identified as the points with the largest contribution to the PC. This information can be retrieved from the columns of the transfer matrix of the associated PCA model. To accurately identify regions associated with recurrent patterns, and filter out spurious points, the geometric information about proximity between the most relevant points is finally taken into account in order to cluster neighbouring relevant points together. This can be achieved in a similar way to finding connected nodes in an undirected graph, and any suitable technique for this task can be employed.

Finally, to be able to estimate the time span of a region of recurrence in a geometric structure, the multi-variate signal can 
be split up into (overlapping) time windows, and the algorithm for spatial detection of recurrence described above can be applied to each window individually. All clusters identified in a window need then to be compared with those found in the previous window, to be able to determine for each cluster whether it is a new cluster or not, or whether a cluster from the previous window has ceased. For the estimate of the time interval of a region of recurrence, we used the first and last window where the region was detected. The mid points of those windows are used to estimate the start and the end of the time interval of recurrence, respectively.

\section{Noninvasive indices for the characterization of recurrence in a patient}

Based on the output of the algorithm for spatio-temporal detection of recurrence, we defined a set of parameters with the aim of noninvasively assessing the type and amount of spatio-temporal recurrence in a patient affected by AF:

- Number of clusters $\left(N_{c}\right)$, describing the number of clusters detected in a patient.

- Median cluster size $(m C S)$, describing the median size of all clusters identified in a patient.

- Median cluster duration $(m C D)$, describing the median duration of all clusters identified in a patient.

- Cluster location $(C L)$, binary variables describing whether there is a cluster in the following locations: front right ( $C L$-FR), front left ( $C L-\mathrm{FL})$, back right ( $C L-\mathrm{BR})$, and black left ( $C L-\mathrm{BL})$.

- Cluster orientation and distribution in the horizontal plane. For each cluster it is counted how many electrodes per vertical column (as in Fig. 1) are in that cluster, flattening the spatial information over the superior/inferior axis. Next the vertical columns for the front and back electrodes are uniformly spread over a semi-circle, such that each electrode in a cluster is represented by a vector in the horizontal plane. After summing the vectors of all electrodes in a cluster, a vector representing the clusterorientation is obtained. From that the following features are derived:

- Resultant angle $(R A)$ : overall angle obtained after summing all cluster-orientations of a patient. Highlights general direction for a patient.

- Resultant Magnitude $(R M)$ : overall magnitude obtained after summing all cluster-orientations of a patient. Represents inter-cluster coherence

- Mean angle $(m A)$ : unweighted average of clusterorientation of the patient; expresses cluster coherence.

- Mean difference in angle among clusters $(m D A)$ : average angular distance between angles of clusterorientation of a patient

Therefore, some indices focus more on the number and duration of recurrence clusters, while others more on the cluster locations. Excluded low-quality leads (Sec. II-A) were not used to compute those indices.
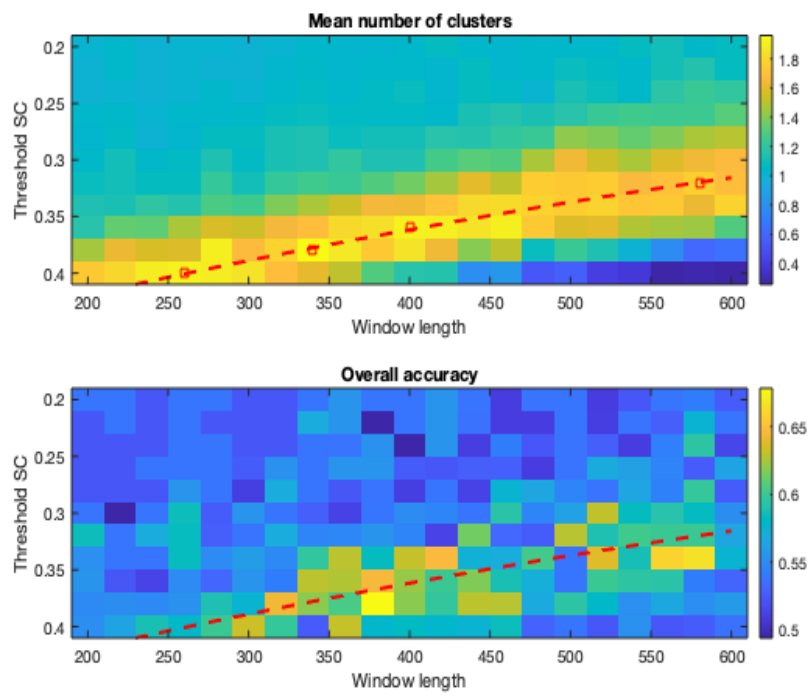

Fig. 2: Grid search on parameter settings. Top: average number of clusters observed over the 74 patients. Bottom: prediction accuracy of overall model on patient outcome. The red dashed line indicates the fit of second order polynomial between window length and threshold for obtaining high average number of clusters.

\section{Analyses}

1) Optimal parameter setting: The algorithm for spatiotemporal detection of recurrence requires a set of parameters to be manually provided. Among those, the window length and the threshold for the SC. Therefore, we ran a grid search to find optimal values for these two parameters, given the data at hand. Optimality was defined in terms of number of clusters identified and separability of recurrent vs. nonrecurrent patients. For this, the window length was varied in the interval $[200,600]$, with a step of 20 , and the threshold of the SC was varied in the interval $[0.2,0.4]$, with step size of 0.02 . The results in this analysis are shown in Fig. 2. Since the goal is to detect recurrence, a high mean number of clusters is beneficial. The value for the threshold for the SC is clearly dependent on the choice of window length by a near linear relation. The optimal fit found for a second order polynomial is $y=0.4881-3.7313 \cdot 10^{-4} x+1.4320 \cdot 10^{-7} x^{2}$.

2) Relation of noninvasive indices of spatio-temporal recurrence with AF recurrence after electrical cardioversion: After identifying the optimal values for the parameter settings, we applied the algorithm for detection of spatio-temporal recurrence to the data introduced in II-A and computed the indices introduced in II-C for all patients. A univariate logistic regression model was then generated for each index to assess their association with AF recurrence after electrical cardioversion. Moreover, a multivariable logistic regression model was also generated with all significant indices as input. Although prediction of treatment outcome and patient stratification were outside the scope of this study, to provide a tentative indication 
TABLE I: Noninvasive indices of recurrence. Numbers are given as median(25th-75th percentile), or count. * times $10^{3}$.

\begin{tabular}{|c|c|c|c|}
\hline Index & AF Recurrence & No AF Recurrence & $p$-value \\
\hline$N_{c}$ & $1.00(1.00)$ & $2.00(2.00)$ & $p<0.031$ \\
$m C S$ & $42.00(43.38)$ & $26.50(83.88)$ & $p=0.1880$ \\
$m C D$ & $0.38(2.02)^{*}$ & $0.38(2.04)^{*}$ & $p=0.9230$ \\
$C L$-FR & 30 & 30 & $p=0.4723$ \\
$C L$-FL & 29 & 30 & $p=1$ \\
$C L$-BR & 20 & 30 & $p<0.004$ \\
$C L$-BL & 24 & 29 & $p=0.1493$ \\
$R A$ & $1.56(0.35)$ & $1.59(0.24)$ & $p=0.7461$ \\
$R M$ & $2.23(1.08)^{*}$ & $2.03(1.11)^{*}$ & $p=0.5927$ \\
$m A$ & $1.55(0.57)$ & $1.50(0.48)$ & $p=0.5449$ \\
$m D A$ & $6.28(5.66)$ & $0.70(5.83)$ & $p=0.0541$ \\
\hline
\end{tabular}

of the added value in a clinical setting, we also assessed overall accuracy of the significant parameters in discriminating between recurrent and non-recurrent patients.

\section{RESULTS}

\section{A. Optimal parameter setting}

The grid search gave the following optimal values for the window length and the threshold for the SC: a window length of 380 samples $(\approx 1.5 \mathrm{sec}$.) and a threshold for the SC of 0.38 . Since for 10 out of 75 patients the threshold 0.38 did not provide any recurrence cluster, in these patients the threshold was set to 0.25 .

B. Relation of noninvasive indices of spatio-temporal recurrence with AF recurrence after electrical cardioversion

Table I provides a summary of statistical differences in the noninvasive indices of recurrence for the patients experiencing AF recurrence 4 to 6 weeks after electrical cardioversion (AF Recurrence) and those who do not (No AF Recurrence). Statistical differences were assessed by means of Wilcoxon sum-rank test for continuous variables, and by means of test for proportions for count variables.

When building a logistic regression model, the univariate analysis showed that only the number of clusters $\left(N_{c}\right)$ and the cluster location back-right $(C L$-BR) were significantly associated with AF recurrence 4 to 6 weeks after electrical cardioversion ( $p=0.0221$ and $p=0.0075$, respectively). The multivariate analysis on the two significant indices showed that only the cluster location back-right remained significantly associated with AF recurrence after electrical cardioversion $(p<0.0186)$.

Fig. 3 shows the median location of clusters of recurrence for the patients showing AF recurrence after electrical cardioversion (top) and those who do not (bottom).

\section{DISCUSSION AND CONCLUSIONS}

In this study, we investigated whether the framework proposed in [14] for detection of spatio-temporal recurrence in complex dynamical systems [14], could be used to identify recurrence of AF propagation patterns noninvasively from BSPM recordings from persistent AF patients. Results showed that clusters of recurrence can be identified on the body surface
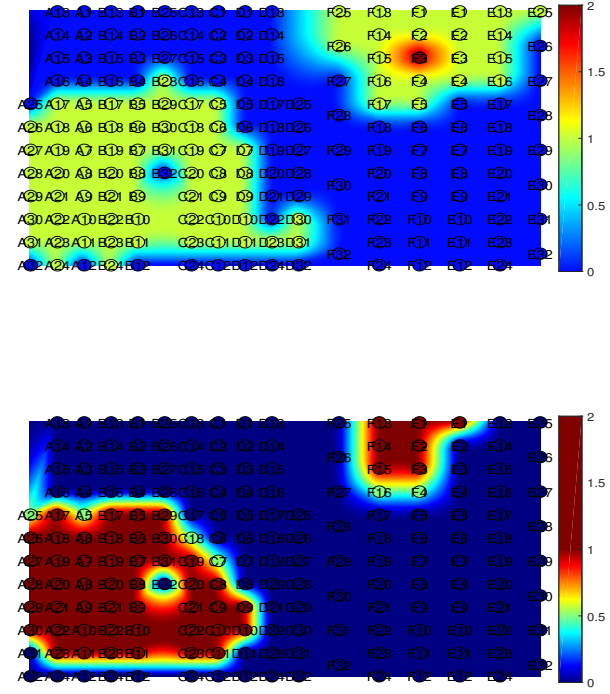

Fig. 3: Median location of clusters of recurrence for the patients showing AF recurrence after electrical cardioversion (top) and those who do not (bottom).
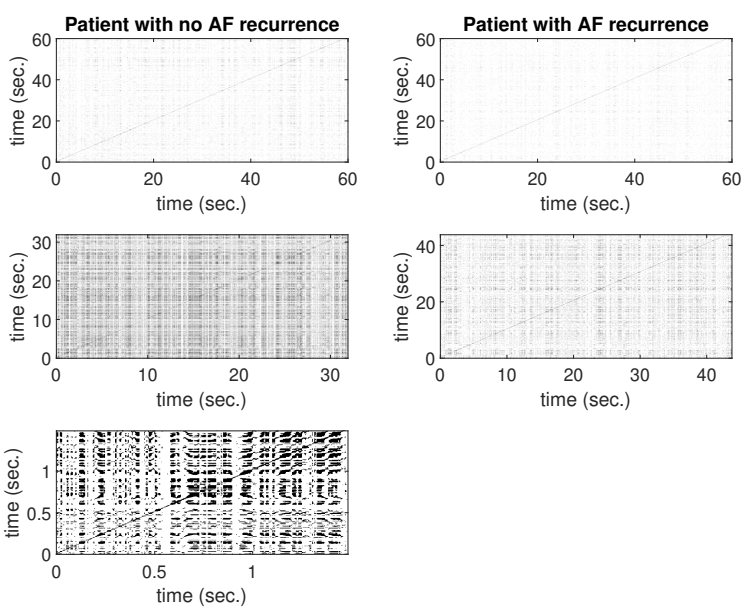

Fig. 4: Right: multivariate recurrence plots from a patient with no AF recurrence 4 to 6 weeks after electrical cardioversion. Left: multivariate recurrence plots from a patient with $\mathrm{AF}$ recurrence.

of patients affected by persistent AF, although the sensitivity is relatively low, with a mean of about 1.5 clusters per patient. Moreover, approximate location of those clusters could be determined, with clusters mainly located bottom-right on the front, and top-left on the back of the torso. When focusing on outcome of electrical cardioversion 4 to 6 weeks after the procedure, only two of the proposed indices of recurrence showed to be significantly associated with AF recurrence after electrical cardioversion. Namely, the number of detected 


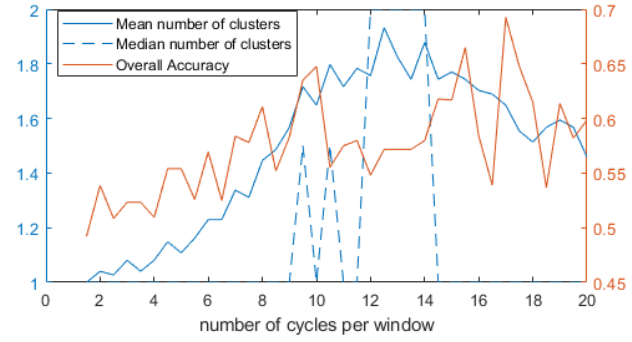

Fig. 5: Number of clusters and accuracy when using a window length that contains a certain number of AF cycles. SC threshold chosen according to fit in Fig. 2.

clusters and the location of some of those clusters top-right on the back of the torso. In Fig. 3 it can be noticed that patients that do not recur after electrical cardioversion show in average larger clusters than patients that recur, with recurrent activity also manifesting on the top-right of the back of the torso, while being completely absent in patients that recur. Overall, this suggests that patients that do not recur are characterized by more organized (more periodic) AF propagation patterns in the atria, which reflects on more recurrent activity and in turn on more and wider recurrence clusters detected on the body surface. This may be explained by a lower degree of electro-structural remodeling in these patients, and thus a less complex AF substrate, which is in line with previous findings on the relation between AF substrate complexity and treatment outcome [11]. This is qualitatively confirmed by the multivariate recurrence plots in Fig. 4 [14], for a patient with no $\mathrm{AF}$ recurrence (right) and a patient with $\mathrm{AF}$ recurrence (left) after electrical cardioversion. The recurrence plots from all leads are shown at the top for each patient (for the entire duration of the recordings), while the plots below it show the recurrence plots only from the leads in each identified cluster (for the time span of the cluster; notice that the number of clusters identified in each patient may differ). It can be noticed how information about recurrence can be better retrieved when focusing on individual clusters, and how recurrence is much more evident in the patient with no AF recurrence after electrical cardioversion.

To detect clusters of recurrence, we used a fixed window length and a fixed threshold of the SC. Optimal values for the data set at hand were obtained by a grid search over a range of plausible values. As we noticed that a fix value of 0.38 for the threshold did not give any cluster for some of the patients, we decided to lower the threshold to 0.25 in these patients. This choice was motivated by the fact that we assumed to be incorrect to conclude that a patient did not present any recurrence cluster, if a cluster could be detected by lowering the threshold for the SC. This also indicates the need for a patient-specific selection of the window length and the threshold for the SC. Based on the relation highlighted in Fig. 2 , we made some attempts to make the selection of the window length and threshold patient-specific, by selecting the window length based on the dominant frequency of AF in a patient. A search on a window length that includes a certain amount of AF cycle lengths, and the threshold of the SC is determined by the fit in Fig. 2, was performed. Preliminary results in Fig. 5 show that the desirable parameters, being number of clusters and accuracy are not in clear agreement when choosing an adaptive window length. This should be thoroughly investigated in a future study. Finally, preliminary results suggest that noninvasive characterization of recurrence of AF propagation patterns in persistent AF patients is possible, and is associated with AF recurrence after electrical cardioversion.

\section{REFERENCES}

[1] G. Lee, S. Kumar, A. Teh, A. Madry, S. Spence, L. Larobina, J. Goldblatt, R. Brown, V. Atkinson, S. Moten, J. Morton, P. Sanders, P. Kistler, and J. Kalman, "Epicardial wave mapping in human long-lasting persistent atrial fibrillation: Transient rotational circuits, complex wavefronts, and disorganized activity," European Heart Journal, vol. 35, no. 2, pp. 86-97, 2014.

[2] M. Allessie, K. Konings, C. Kirchhof, and M. Wijffels, "Electrophysiologic mechanisms of perpetuation of atrial fibrillation," Am J Cardiol, vol. 77, no. 3, pp. 10A-23A, 1996.

[3] M. Allessie, N. de Groot, R. Houben, U. Schotten, E. Boersma, J. Smeets, and H. Crijns, "Electropathological substrate of long-standing persistent atrial fibrillation in patients with structural heart disease: longitudinal dissociation," Circ Arrhythm Electrophysiol, vol. 3, no. 6, pp. 606-615, 2010.

[4] J. Jalife, "Déjà vu in the theories of atrial fibrillation dynamics," Cardiovascular Research, vol. 89, no. 4, pp. 766-775, 2010.

[5] J.-P. Eckmann, S. Oliffson Kamphorst, and D. Ruelle, "Recurrence Plots of Dynamical Systems," Europhys. Lett., vol. 4 (9), pp. 973-977, 1987.

[6] J. Ng, D. Gordon, R. S. Passman, B. P. Knight, R. Arora, and J. J. Goldberger, "Electrogram morphology recurrence patterns during atrial fibrillation," Heart Rhythm, vol. 11, no. 11, pp. 2027 - 2034, 2014.

[7] S. Zeemering, P. Bonizzi, B. Maesen, R. Peeters, and U. Schotten, "Recurrence quantification analysis applied to spatiotemporal pattern analysis in high-density mapping of human atrial fibrillation," in Conf Proc IEEE Eng Med Biol Soc, 2015, pp. 7704-7.

[8] T. A. R. Lankveld, S. Zeemering, H. J. G. M. Crijns, and U. Schotten, "The ECG as a tool to determine atrial fibrillation complexity," Heart, vol. 100, pp. 1077-84, 2014.

[9] U. Schotten, B. Maesen, and S. Zeemering, "The need for standardization of time- and frequency-domain analysis of body surface electrocardiograms for assessment of the atrial fibrillation substrate," EP Europace, vol. 14, no. 8, pp. 1072-1075, 2012.

[10] P. Bonizzi, S. Zeemering, J. M. Karel, L. Y. Di Marco, L. Uldry, J. Van Zaen, J. M. Vesin, and U. Schotten, "Systematic comparison of non-invasive measures for the assessment of atrial fibrillation complexity: a step forward towards standardization of atrial fibrillation electrogram analysis," Europace, vol. 17(2), pp. 318-25, 2015 Feb.

[11] S. Zeemering, T. Lankveld, P. Bonizzi, I. Limantoro, S. Bekkers, H. Crijns, and U. Schotten, "The electrocardiogram as a predictor of successful pharmacological cardioversion and progression of atrial fibrillation," EP Europace, vol. 20, no. 7, pp. e96-e104, 072018.

[12] O. Meste, S. Zeemering, J. Karel, T. Lankveld, U. Schotten, H. Crijns, R. Peeters, and P. Bonizzi, "Noninvasive Recurrence Quantification Analysis Predicts Atrial Fibrillation Recurrence in Persistent Patients Undergoing Electrical Cardioversion," in Proc. Computing in Cardiology, no. 43, 2016, pp. 677-680.

[13] P. Bonizzi, S. Zeemering, J. Karel, M. Azman, T. Lankveld, U. Schotten, H. Crijns, R. Peeters, and O. Meste, "Noninvasive Characterisation of Short- and Long-Term Recurrence of Atrial Signals During Persistent Atrial Fibrillation," In Proc. Computing in Cardiology 2017; 44.

[14] P. Bonizzi, R. Peeters, S. Zeemering, A. van Hunnik, O. Meste, and J. Karel, "Detection of spatio-temporal recurrent patterns in dynamical systems," Frontiers in Applied Mathematics and Statistics, vol. 5, p. 36, 2019.

[15] R. Alcaraz and J. J. Rieta, "Adaptive singular value cancellation of ventricular activity in single-lead atrial fibrillation electrocardiograms," Physiol. Meas., vol. 29, pp. 1351-1369, 2008. 